\title{
TWO THEOREMS ON MULTIPLE INTEGRALS *
}

BY PHILIP FRANKLIN

1. Introduction. The multiple integrals in question here were discussed by Poincaré (Acta Mathematica, vol. 9, p. 321) in a paper in which he defined them and derived their integrability conditions. In this note we give two theorems, which simplify the derivation of these conditions, and enable us to express the integral of an integrable function over an open region as an integral of one order lower over the boundary of this region. The second theorem was suggested by a special case proved by Professor Eisenhart.

2. First Theorem. Our first theorem is

$$
\begin{aligned}
\int n \int \sum A_{i_{1} \ldots i_{n}} d x_{i_{1}} \cdots d x_{i_{n}} \\
\quad \frac{1}{m-n} \int n+1 \int \sum \frac{\partial A_{i_{1} \ldots i_{n}}}{\partial x_{i_{n+1}}} d x_{i_{n+1}} d x_{i_{1}} \cdots d x_{i_{n}},
\end{aligned}
$$

where the $n$ (dimensional) region of integration for the left member is the boundary of the $n+1$ region for the right member, and the summations apply to all $n$ or $n+1$ permutations of the $m$ subscripts of our fundamental space. The theorem is proved by adding equations of the form

$$
\begin{aligned}
\int n \int A_{1 \ldots n} d x_{1} \cdots & d x_{n} \\
& =\int n+1 \int \frac{\partial A_{1 \ldots n}}{\partial x_{n+1}} d x_{n+1} d x_{1} \cdots d x_{n},
\end{aligned}
$$

which may be established when the $A$ 's are functions of $m$ variables by methods similar to those ordinarily used to prove (2) in $n$-space. The equation (1) shows that the set of equations

$$
\sum_{j}(-1)^{j-1} \frac{\partial A_{i_{1} \ldots i_{j-1} i_{j+1} \cdots i_{n+1}}}{\partial x_{i_{j}}}=0
$$

is a necessary and sufficient condition for the vanishing of the left member of (1) for all closed regions of integration, i.e.,

\footnotetext{
* Presented to the Society, September 7, 1922.
} 
that (3) is the condition of integrability for the form

$$
\sum A_{i_{1} \ldots i_{n}} d x_{i_{1}} \cdots d x_{i_{n}} .
$$

3. Second Theorem. It follows from (1) also that the integral

$$
\int n+1 \int \sum B_{i_{1} \ldots i_{n+1}} d x_{i_{1}} \cdots d x_{i_{n+1}},
$$

taken over any open region may be expressed as an integral

$$
(m-n)(n+1) \int n \int \sum A_{i_{1} \ldots i_{n}} d x_{i_{1}} \cdots d x_{i_{n}},
$$

taken over its boundary, provided $A$ 's can be found such that

$$
B_{i_{1} \ldots i_{n+1}}=\sum_{j}(-1)^{j-1} \frac{\partial A_{i_{1} \ldots i_{j-1} i_{j+1} \ldots i_{n+1}}}{\partial x_{j}} .
$$

We shall show that a necessary and sufficient condition for the existence of such a set of $A$ 's is that (5) be integrable, i.e., that

$$
\sum(-1)^{j-1} \frac{\partial B_{i_{1} \ldots i_{j-1} i_{j+1} \ldots i_{n+2}}}{\partial x_{j}}=0 .
$$

This theorem is known for the case $n=0$. We shall outline an induction by which the general case may be reduced to this one. We first consider the case where there are $n+2 i$ 's, and hence $n+2$ equations (7) and one condition (8). If we write (9) $B_{1 \ldots p-1, p+1 \ldots n+2}=B_{1 \ldots p-1, p+1 \ldots n+1}^{\prime}$

$$
+(-1)^{n} \frac{\partial A_{1 \ldots p-1, p+1 \ldots n+1}}{\partial x_{n+2}},
$$

where $p \neq n+2$, and where the $B^{\prime}$ s are defined by the corresponding equation (7), and if we determine the $A$ 's appearing explicitly in (9) so as to satisfy the remaining equation of the set (7),

$$
B_{1 \ldots n+1}=\sum_{j}(-1)^{j-1} \frac{\partial A_{1 \ldots j-1, j+1 \ldots n+1}}{\partial x_{j}},
$$

but leave their values otherwise arbitrary, we find that by the use of (9) and (10) the condition (8) reduces to the form

$$
\sum_{j}(-1)^{j-1} \frac{\partial B_{1 \ldots j-1, j+1 \ldots n+1}^{\prime}}{\partial x_{j}}=0 .
$$

Since (11) is of the same form as (8), but in one less index, 
and since the expressions for the $B$ 's in terms of $A$ 's are similar to (7), we have reduced the problem to one of lower order. To complete our proof, we reduce the case for $m+1$ subscripts to that for $m$. We first obtain values for the $A$ 's not involving one subscript, say $k$, from the equations and conditions not involving $k$, by the case assumed as the basis of the induction. Then we make the substitutions:

$$
B_{i_{1} \ldots i_{n} k}=B_{i_{1} \ldots i_{n}}^{\prime}+(-1)^{n} \frac{\partial A_{i_{1} \ldots i_{n}}}{\partial x_{k}},
$$

which effects the desired reduction.

This proves the sufficiency of the conditions; that they are necessary follows by direct substitution. A more complete discussion of the properties of multiple integrals is given in an expository article that will appear in the ANNALS OF Mathematics.

Harvard Universtty

\section{KIRKMAN PARADES *}

BY F. N. COLE

On examining the complete list of non-equivalent triad systems in 15 letters published in the Memoirs of the NationaL ACademy of ScIences (vol. 14, No. 2, pp. 77-80), it appears that only four of these are Kirkman systems, if this name be applied to those cases where the 35 triads divide into seven sets (or columns) of five with each column containing all the 15 letters. Such a seven-column arrangement might be called a Kirkman parade. And it turns out that three of the Kirkman systems give each two non-equivalent parades, while the fourth system gives only one parade.

Kirkman proposed his problem in the Lady's and GentleMAN's DiARY for 1850 . The seven solutions were correctly given by Woolhouse in the same Diary for 1862 and 1863. In 1881 Carpmael published a list of eleven solutions in the Proceedings of the London Mathematical Society (vol. 12, pp. 148-156). But his sixth and seventh items duplicate the third and fourth, and the fifth and eleventh duplicate the ninth and tenth.

\footnotetext{
* Presented to the Society, September 7, 1922.
} 